\title{
Una nueva noción de conjuntos neutrosóficos a través de los conjuntos *b-abiertos en espacios topológicos neutrosóficos
}

\section{A new notion of neutrosophic sets through neutrosophic *b-open sets in neutrosophic topological spaces}

\author{
Carlos Andres Granados-Ortiz \\ aEstudiante de Doctorado en Matemáticas, carlosgranadosortiz@outlook.es, https://orcid.org/0000-0002-7754-1468, Universidad de Antioquia, Medellín, \\ Colombia
}

Forma de citar: Granados-Ortiz, C. A.. (2021). Una nueva noción de conjuntos neutrosóficos a través de los conjuntos *b-abiertos en espacios topológicos neutrosóficos. Eco Matemático, 12 (2), 54-64

Recibido: 2 de marzo de 2021

Aceptado: 29 de abril de 2021

\section{Palabras clave \\ Conjuntos neutrosóficos \\ *b-abiertos, conjuntos neutrosóficos \\ *b-cerrados, operador neutrosófico \\ *b-interior, operador neutrosófico \\ *b-clausura.}

Resumen: La idea principal de este artículo es introducir una nueva noción asociada a los espacios topológicos neutrosóficos y mostrar algunos resultados teniendo en cuenta estas nociones. La noción que se está introduciendo es la noción de los conjuntos neutrosóficos *b-abiertos en espacios topológicos neutrosóficos y se prueba algunas de sus propiedades y caracterizaciones. Adicionalmente, se estudia la noción de operador neutrosófico *b-interior y operador neutrosófico *b-clausura.

*Autor para correspondencia: carlosgranadosortiz@outlook.es

Doi: https://doi.org/10.22463/17948231.3182 


\section{Keywords}

Neutrosophic *b-open

set,

Neutrosophic *b-closed

set,

Neutrosophic *b-

interior operator,

Neutrosophic *b-closure

operator.
Abstract: The main idea of this paper is to introduce a new notion associated to neutrosophic topological space and show some results based on it. The notion that we are introducing is namely *b-open set in neutrosophic topological spaces and we prove some of their properties and characterization. Additionally, we also study neutrosophic *b-interior and neutrosophic *b-closure operators.

\section{Introducción}

La teoría de conjuntos difusos (Zadeth, 1965), la teoría de conjuntos difusos intuicionistas (Atanassov, 1986), la teoría de conjuntos neutrosóficos (Smarandache, 2010) y la teoría de conjuntos neutrosóficos de intervalo (Hanafy, 2003) pueden considerarse herramientas para tratar las incertidumbres. Sin embargo, todas estas teorías tienen sus propias dificultades que señaló Smradache (2010). Zadeth (1965) introdujo la teoría de conjuntos difusos como una herramienta matemática para tratar las incertidumbres en las que cada elemento tenía un grado de pertenencia. El conjunto difuso intuicionista fue introducido por Atanassov (1986) como una generalización del conjunto difuso, donde además del grado de pertenencia y el grado de no pertenencia de cada elemento. El conjunto neutrosófico fue introducido por Smarandache (2010) y, explicado, el conjunto neutrosófico es una generalización del conjunto difuso intuicionista. Salama y Alblowi (2012) introdujo el concepto de espacios topológicos neutrosóficos. Introdujeron el espacio topológico neutrosófico como una generalización del espacio topológico difuso intuicionista y un conjunto neutrosófico además del grado de pertenencia, el grado de indeterminación y el grado de no pertenencia de cada elemento.
Este artículo se divide en cinco secciones. La sección (2) consta de las definiciones básicas y algunas propiedades bien conocidas que se utilizan en las secciones posteriores. La sección (3) trata de la definición de conjunto neutrosófico *b-abierto en espacios topológicos neutrosóficos y sus diversas propiedades. La sección (4) trata de la definición de conjunto neutrosófico *b-cerrado en espacios topológicos neutrosóficos y sus diversas propiedades. La sección (5) trata de los conceptos de operadores neutrosóficos *b-interior y neutrosóficos *b-de clausura y estudiamos algunas de sus propiedades.

Las nociones aquí presentadas se pueden extender a la topológica neutrosófica definiendo generalizaciones (Salama y Albowi, 2012), espacios bitopológicos (Das y Tripathy, 2020), conjuntos neutrosóficos difusos (Karaaslan, 2015), conjuntos neutrosóficos pitagóricos (Granados y Dhital, 2021) y entre otros. Además, utilizando la noción de neutro-algebra definida por Smarandache (2020), se pueden estudiar las nociones aquí presentadas en pentapartitioned (Das et al. 2021). Por otro lado, estas nociones se pueden definir y estudiar en conjuntos neutrosóficos clásicos (Granados, 2020) y en la estadística convergencia (Granados y Dhital, 2021). Por otro lado, sería de gran interés que estas nociones puedan estudiar y aplicarse en TIC y educación matemática para su enseñanza 
en instituciones educativas de nivel superior, para conocer más sobre TIC y educación en las matemáticas ver los trabajos de (Zamora et al., 2020), (Vergel et al., 2016) y (Granados y Padilla, 2021).

\section{Nociones Preliminares}

En esta sección, recordamos algunas definiciones bien conocidas sobre conjuntos neutrosóficos y sus operaciones. Las definiciones y proposiciones presentadas a continuación, fueron definidas por (Salama y Alblowi, 2012).

Definición 2.1 Sea Y un conjunto arbitrario no vacío. Un conjunto neutrosófico $(N S) B$ es un objeto que tiene la $B=\{<y, \mu B(y), o r B(y), \mu B(y)>: y \in Y\}$ donde $\mu B(y), o B(y)$ y $\gamma B(y)$ representan las funciones de grado de membresía, grado de indeterminación y el grado de no membresía de cada elemento $y \in \mathbf{Y}$ al conjunto B.

Observación 2.2 Un conjunto neutrosófico B $=\{<\mathrm{y}, \mu \mathrm{B}(\mathrm{y}), o \mathrm{~B}(\mathrm{y}), \gamma \mathrm{B}(\mathrm{y})>: \mathrm{y} \in \mathrm{Y}\}$ se conoce como una tripleta ordenada $<\mu \mathrm{B}, o \mathrm{~B}, \gamma \mathrm{B}>$ en $]^{-} 0,1^{+}$ [ sobre Y.

Observación 2.3 Durante el desarrollo de este artículo, denotaremos $\mathrm{B}=<\mathrm{y}, \mu_{\mathrm{B}}, o_{\mathrm{B}}, \gamma_{\mathrm{B}}>$ para el conjunto neutrosófico $\mathrm{B}=\{<\mathrm{y}, \mu \mathrm{B}(\mathrm{y}), o \mathrm{~B}(\mathrm{y}), \gamma \mathrm{B}(\mathrm{y})$ $>: y \in Y$ \}.

Ejemplo 2.4 Sea B un conjunto no vacío IFS de $Y$, se puede observar que $\mathrm{B}$ es un conjunto NS de la forma $B=\{<\mathrm{y}, \mu \mathrm{B}(\mathrm{y}), 1-(\gamma \mathrm{B}(\mathrm{y})+\gamma \mathrm{B}(\mathrm{y})), \gamma \mathrm{B}(\mathrm{y})$ $>: \mathrm{y} \in \mathrm{Y}\}$.

Dado que nuestro objetivo principal es construir herramientas para desarrollar conjuntos neutrosóficos y topología neutrosófica, se debe introducir las nociones de $N S 0 \mathrm{~N}$ y $1_{\mathrm{N}}$ en $\mathrm{Y}$ de la siguiente manera:

$0 \mathrm{~N}$ se define de la siguiente manera:
(01) $0 \mathrm{~N}=\{<y, 0,0,1>: y \in \mathrm{Y}\}$

(02) $0 \mathrm{~N}=\{<y, 0,1,1>: y \in \mathrm{Y}\}$

(03) $0 \mathrm{~N}=\{<y, 0,1,0\rangle: y \in \mathrm{Y}\}$

(04) $0 \mathrm{~N}=\{\langle y, 0,0,0\rangle: y \in \mathrm{Y}\}$

$1 \mathrm{~N}$ se define de la siguiente manera:

(11) $1 \mathrm{~N}=\{<y, 1,0,0\rangle: y \in \mathrm{Y}\}$

(12) $1 \mathrm{~N}=\{<y, 1,0,1>: y \in \mathrm{Y}\}$

(13) $1 \mathrm{~N}=\{\langle y, 1,1,0>: y \in \mathrm{Y}\}$

(14) $1 \mathrm{~N}=\{<y, 1,1,1>: y \in \mathrm{Y}\}$

Definición 2.5 Sea $\mathrm{B}=<\mu_{\mathrm{B}}, o_{\mathrm{B}}^{*}, \gamma_{\mathrm{B}}>$ un conjunto $N S$ sobre Y, el complemento del conjunto $B(\mathrm{C}(\mathrm{B}))$ se define de las siguientes maneras: $\mathrm{y} \in \mathrm{Y}\}$

$\left(\mathrm{C}_{1}\right) \mathrm{C}(\mathrm{B})=\left\{<\mathrm{y}, 1-\mu_{\mathrm{B}}(\mathrm{y}), 1-\sigma_{\mathrm{B}}^{*}(\mathrm{y}), 1-\gamma_{\mathrm{B}}(\mathrm{y})>\right.$ :

$\left(\mathrm{C}_{2}\right) \mathrm{C}(\mathrm{B})=\left\{<\mathrm{y}, \gamma_{\mathrm{B}}(\mathrm{y}), o_{\mathrm{B}}^{\prime}(\mathrm{y}), \mu_{\mathrm{B}}(\mathrm{y})>: \mathrm{y} \in \mathrm{Y}\right\}$

$\left(\mathrm{C}_{3}\right) \mathrm{C}(\mathrm{B})=\left\{<\mathrm{y}, \gamma_{\mathrm{B}}(\mathrm{y}), 1-\sigma_{\mathrm{B}}(\mathrm{y}), \mu_{\mathrm{B}}(\mathrm{y})>: \mathrm{y} \epsilon\right.$ Y\}

Teniendo en cuenta la definición 2.5, podemos definir varias relaciones y operaciones entre los conjuntos NSs de la siguiente manera:

Definición 2.6 Sea y be un no vacío, y sean B y D conjuntos neutrosóficos de la forma $B=$ $\{<\mathrm{y}, \mu \mathrm{B}(\mathrm{y}), o \mathrm{~B}(\mathrm{y}), \gamma \mathrm{B}(\mathrm{y})>: \mathrm{y} \in \mathrm{Y}\}$ y $\mathrm{D}=\{<\mathrm{y}$, $\mu \mathrm{D}(\mathrm{y}), o \mathrm{D}(\mathrm{y}), \in \mathrm{D}(\mathrm{y})>: \mathrm{y} \in \mathrm{Y}\}$. Entonces, podemos considerar los siguientes enunciados para definir la noción de subconjuntos $(\mathrm{B} \subseteq \mathrm{D})$ :

(1) $\mathrm{B} \subseteq \mathrm{D} \leftrightarrow \mu \mathrm{B}(\mathrm{y}) \leq \mu_{\mathrm{D}}(\mathrm{y}), o \mathrm{~B}(\mathrm{y}) \leq o \mathrm{D}(\mathrm{y}) \mathrm{y}$ $\gamma \mathrm{B}(\mathrm{y}) \geq o \mathrm{D}(\mathrm{y}) \forall \mathrm{y} \in \mathrm{Y}$

(2) $\mathrm{B} \subseteq \mathrm{D} \leftrightarrow \mu \mathrm{B}(\mathrm{y}) \leq \mu \mathrm{D}(\mathrm{y}), o \mathrm{~B}(\mathrm{y}) \geq o \mathrm{D}(\mathrm{y}) \mathrm{y}$ $\gamma \mathrm{B}(\mathrm{y}) \geq \gamma \mathrm{D}(\mathrm{y}) \forall \mathrm{y} \in \mathrm{Y}$

Proposición 2.7 Para cualquier conjunto neutrosófico $\mathrm{B}$, los siguientes enunciados se satisfacen:

(1) $0_{\mathrm{N}} \subseteq B, 0_{\mathrm{N}} \subseteq 0_{\mathrm{N}}$

(2) $B \subseteq 1_{\mathrm{N}}, 1_{\mathrm{N}} \subseteq 1_{\mathrm{N}}$ 
Definición 2.8 Sea Y un conjunto no vacío, y sean $\mathrm{B}=<\mathrm{y}, \mu \mathrm{B}(\mathrm{y}), o \mathrm{~B}(\mathrm{y}), \gamma \mathrm{B}(\mathrm{y})>, \mathrm{D}=<\mathrm{y}, \mu \mathrm{D}(\mathrm{y})$, $o \mathrm{D}(\mathrm{y}), \gamma \mathrm{D}(\mathrm{y})>$ conjuntos NSs. Entonces,

(1) $B \cap D$ se puede definir de la siguiente manera:

$\left(\mathrm{I}_{1}\right) B \cap D=\left\langle y, \mu_{B}(y) \wedge \mu_{D}(y), \sigma_{B}(y) \wedge \sigma_{D}(y)\right.$ y $\left.\gamma_{B}(y) \vee \gamma_{D}(y)\right\rangle$

$\left(\mathrm{I}_{2}\right) B \cap D=\left\langle y, \mu_{B}(y) \wedge \mu_{D}(y), \sigma_{B}(y) \vee \sigma_{D}(y)\right.$ y $\left.\gamma_{B}(y) \vee \gamma_{D}(y)\right\rangle$

(2) $B \cup D$ se puede definir de la siguiente manera:

$\left(U_{1}\right) B \cup D=\left\langle y, \mu_{B}(y) \vee \mu_{D}(y), \sigma_{B}(y) \vee \sigma_{D}(y)\right.$ y $\left.\gamma_{B}(y) \wedge \gamma_{D}(y)\right\rangle$

$\left(\cup_{2}\right) B \cup D=\left\langle y, \mu_{B}(y) \vee \mu_{D}(y), \sigma_{B}(y) \wedge \sigma_{D}(y) \mathrm{y} \gamma_{B}(y) \wedge \gamma_{D}(y)\right\rangle$

Podemos generalizar fácilmente las operaciones de intersección y unión en la Definición 2.8 a una familia arbitraria de NS de la siguiente manera:

Definición 2.9 Sea $\{B j: j \in J\}$ una familia arbitraria de conjuntos NS en Y, entonces

(1) $\cap B_{j}$ se puede definir de la siguiente manera:

(i) $\cap B_{j}=\left\langle y, \bigwedge_{\mathrm{j} \in \mathrm{J}} \mu(y), \bigwedge_{\mathrm{j} \in \mathrm{J}} \sigma(y), \bigvee_{\mathrm{j} \in \mathrm{J}} \gamma(y)\right\rangle$

(ii) $\cap B_{j}=\left\langle y, \Lambda_{\mathrm{j} \in \mathrm{J}} \mu(y), \bigvee_{\mathrm{j} \in \mathrm{J}} \sigma, \bigvee_{\mathrm{j} \in \mathrm{J}} \gamma(y)\right\rangle$

(2) $\cup B_{j}$ se puede definir de la siguiente manera:

(i) $\cup B_{j}=\langle y, \mathrm{~V}, \mathrm{~V}, \wedge\rangle$

(ii) $\cup B_{j}=\langle y, \vee, \wedge, \wedge\rangle$

Proposición 2.10 Para todo conjunto neutrosófico B y D, los siguientes enunciados se satisfacen:

(1) $\mathrm{C}(\mathrm{B} \cap \mathrm{D})=\mathrm{C}(\mathrm{B}) \cap \mathrm{C}(\mathrm{D})$

(2) $\mathrm{C}(\mathrm{B} \cap \mathrm{D})=\mathrm{C}(\mathrm{B}) \cap \mathrm{C}(\mathrm{D})$.

Aquí extendemos los conceptos de espacio topológico difuso (Chang, 1968) y espacio topológico difuso intuicionista (Coker, 1997) y al caso de los conjuntos neutrosóficos (Smarandache, 2002):

Definición 2.11 La topología neutrosófica (NT) de un conjunto $\mathrm{Y}$ es una familia $\tau$ de subconjuntos neutrosóficos en $\mathrm{X}$ que satisfacen las siguientes condiciones:

(NT1) $0_{\mathrm{N}}, 1_{\mathrm{N}} \in \tau$,

(NT2) $\mathrm{W}_{1} \cap \mathrm{W}_{2} \in \tau$ para cualquier $\mathrm{W}_{1}, \mathrm{~W}_{2} \in \tau$, (NT3) $\mathrm{U} \mathrm{W}_{\mathrm{i}} \in \tau$ para todo $\left\{\mathrm{W}_{\mathrm{i}}: \mathrm{i} \in \mathrm{J}\right\} \subseteq \tau$

En este caso, el par $(\mathrm{Y}, \tau)$ es un espacio topológico neutrosófico (NTS). Los elementos de $\tau$ son llamados conjuntos abiertos neutrosóficos $(N O S)$. Por otro lado, un conjunto neutrosófico E es cerrado si y solo $\mathrm{C}(\mathrm{E})$ es un conjunto neutrosófico abierto.

Ejemplo 2.12 Cualquier espacio topológico difuso $\left(\mathrm{Y}, \tau_{0}\right)$ en el sentido de Chang es un NTS de la forma $\tau=\left\{\mathrm{B}: \mu \mathrm{B} \in \tau_{0}\right\}$ dondequiera que se identifique un conjunto difuso en $\mathrm{Y}$ cuya función de pertenencia es $\mu \mathrm{B}$ con sus contrapartes.

Observación 2.13 Los espacios topológicos neutrosóficos son generalizaciones muy naturales de los espacios topológicos difusos que permiten que funciones más generales sean miembros de la topología difusa.

$$
\begin{aligned}
& \text { Ejemplo 2.14 Sea } Y=\{y\} y \\
& P=\{<y, 0.5,0.5,0.4>: y \in Y\} \\
& L=\{<x, 0.4,0.6,0.8>: y \in Y\} \\
& M=\{<x, 0.5,0.6,0.4>: y \in Y\} \\
& N=\{<x, 0.4,0.5,0.8>: y \in Y\}
\end{aligned}
$$

Entonces, la familia $\tau=\left\{0_{\mathrm{N}}, P, L, M, N, 1_{\mathrm{N}}\right\}$ de $N S$ en $\mathrm{Y}$ es una neutrosófica topología sobre Y.

Definición 2.15 El complemento de $B(C(B))$ de $N O S$ es un conjunto neutrosófico cerrado $N C S$ en $\mathrm{Y}$. 
Ahora, definimos la clausura neutrosófica y el interior neutrosófico en los espacios topológicos neutrosóficos:

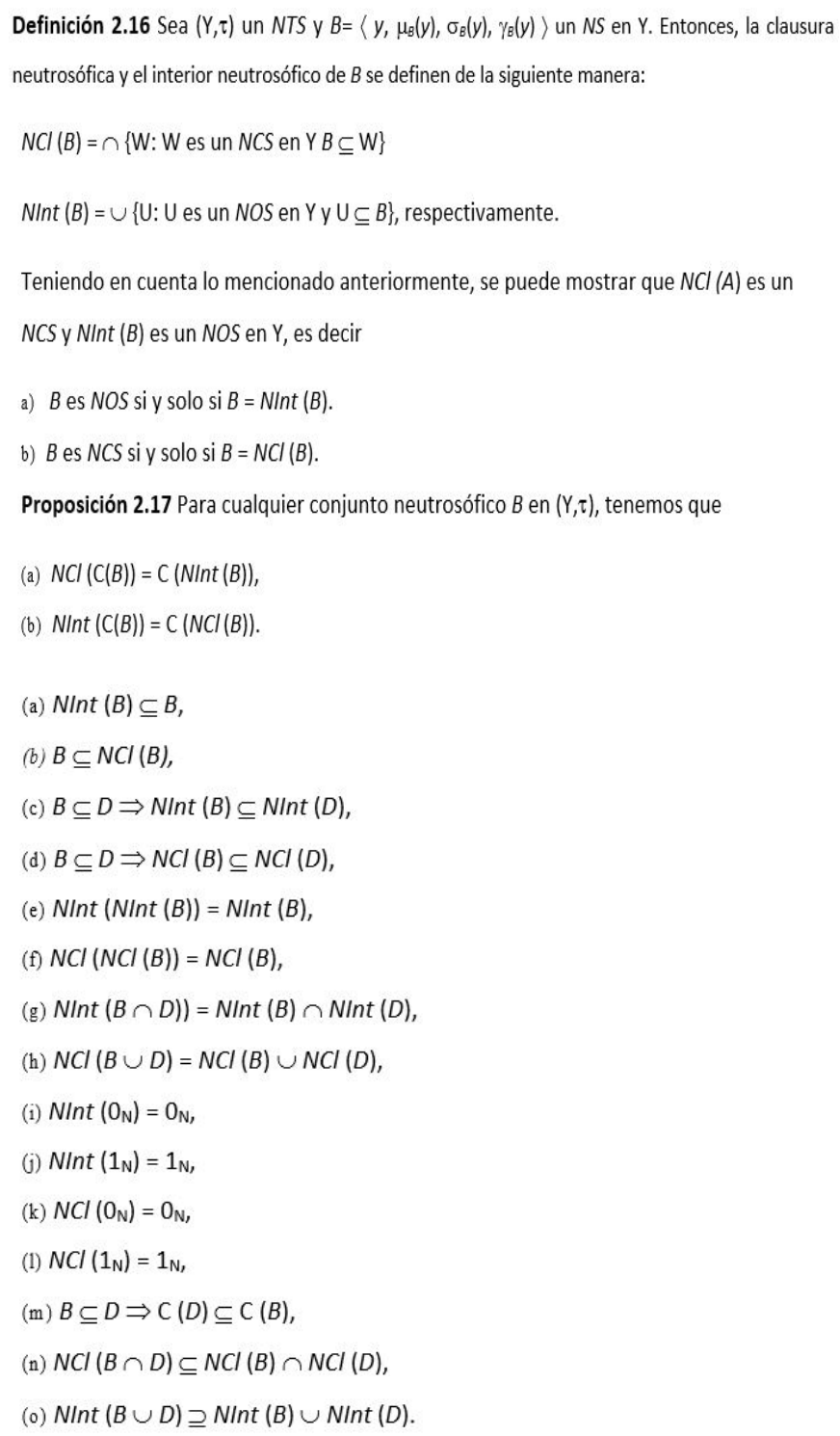

\section{Conjuntos neutrosóficos *b-abiertos en espacios topológicos neutrosóficos}

En esta sección, se introduce la noción del conjunto neutrosófico *b-abierto y también se discuten sus propiedades y caracterizaciones.
Definición 3.1 Sea B un NS de un NTS Y. Entonces, $B$ es un conjunto neutrosófico *b-abierto $\left(N^{*} \mathrm{bO}\right)$ de $\mathrm{Y}$ si $B \subseteq N C l(\mathrm{~B}) \cap N I n t$ (B). El complemento de un conjunto neutrosófico *b-abierto es un conjunto neutrosófico *b-cerrado y se denotará como $N^{*} \mathrm{bC}$.

El siguiente teorema es una caracterización de los conjuntos $N^{*}$ bO en NTS.

Teorema 3.2 Sea $(\mathrm{Y}, \tau)$ un NTS. Entonces, la unión de dos conjuntos $N^{*}$ bO es un conjunto $N^{*}$ bO en NTS Y.

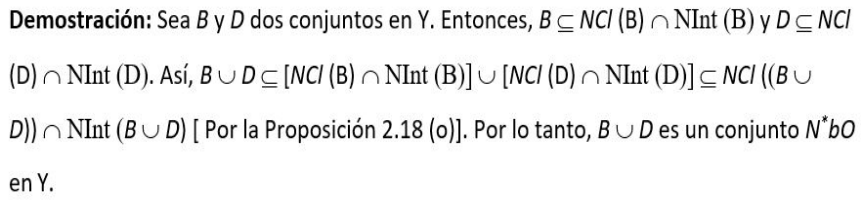
en Y.

Teorema 3.3 Sea $(Y, \tau)$ un NTS. Si $\left\{\mathrm{B}^{\infty}\right\}_{\mathrm{\aleph}_{\in A}}$ es una colección de conjuntos $\mathrm{N}^{*} \mathrm{bO}$ en un NTS Y. Entonces, $\mathrm{U}_{\alpha_{\in \in}} \mathrm{B}_{\alpha}$ es $\boldsymbol{N} \boldsymbol{*} \boldsymbol{b} \boldsymbol{O}$ en $\mathrm{Y}$.

Demostración: La demostración se sigue directamente del teorema 3.2.

Observación 3.4 La intersección de dos $N^{*} b O$ no necesariamente es un conjunto $N^{*} b O$ en $\mathrm{Y}$ como se puede ver en el siguiente ejemplo.

$$
\begin{aligned}
& \text { Ejemplo 3.5 Sea } \mathrm{Y}=\{q, w\} \text { y } \\
& \begin{array}{l}
\mathrm{P}=<(0.4,0.1,0.8),(0.3,0.2,0.5)> \\
\mathrm{F}=<(0.5,0.1,0.8),(0.2,0.4,0.1)> \\
\mathrm{G}=<(0.7,0.5,0.1),(0.6,0.6,0.1)> \\
\mathrm{H}=<(0.9,0.2,0.7),(0.5,0.5,0.5)>
\end{array}
\end{aligned}
$$

Entonces $\tau=\left\{0_{\mathrm{N}}, \mathrm{P}, \mathrm{F}, \mathrm{G}, \mathrm{H}, 1 \mathrm{~N}\right\}$ es NTS sobre Y. Ahora, definamos los siguientes conjuntos $N^{*} b O$ de la siguiente manera:

$$
\begin{array}{r}
\mathrm{P} 1=<(0.4,0.6,0.4),(0.8,0.3,0.4)>\text { y } \mathrm{P} 2= \\
<(1,0.9,0.2),(0.5,0.7,0)>. \text { Podemos ver que } \mathrm{P} 1
\end{array}
$$


$\cap \mathrm{P} 2=<(0.4,0.6,0.4),(0.5,0.3,0.4)>$ no es un conjunto $\mathrm{N}^{*} \mathrm{bO}$ en $\mathrm{Y}$.

Teorema 3.6 Todo conjunto neutrosófico abierto en NTS Y es un conjunto $\mathrm{N}^{*} \mathrm{bO}$ en $\mathrm{Y}$.

Demostración: La demostración se sigue teniendo en cuenta la definición de $\mathrm{N}^{*} \mathrm{bO}$.

Observación 3.7 El reciproco del teorema anterior no se cumple de manera general como se puede ver en el siguiente ejemplo.

Ejemplo 3.8 Sea $\mathrm{Y}=\{q, w, e\}$ con $\tau=\{0 \mathrm{~N}$, $\mathrm{P}, \mathrm{O}, 1 \mathrm{~N}\}$. Algunos de los conjuntos $\mathrm{N} * \mathrm{bO}$ de $(\mathrm{Y}, \tau$ son: $0.8)>$

$$
\mathbf{P}=<(0.4,0.5,0.2),(0.3,0.2,0.1),(0.9,0.6
$$

$\mathbf{O}=<(0.2,0.4,0.5),(0.1,0.1,0.2),(0.6,0.5$ $0.8)>$ $0.5)>$

$\mathbf{Q}=<(0.5,0.6,0.1),(0.4,0.3,0.1),(0.9,0.8$

$\mathbf{S}=<(0.3,0.5,0.4),(0.1,0.6,0.2),(0.7,0.5$ $0.8)>$

$$
\mathbf{F}=<(0.5,0.6,0.1),(0.4,0.6,0.1),(0.9,0.8
$$
$0.5)>$ $0.8)>$

$\mathbf{G}=<(0.3,0.5,0.4),(0.1,0.3,0.2),(0.7,0.5$,

$$
\mathbf{J}=<(0.4,0.5,0.2),(0.3,0.6,0.1),(0.9,0.6,
$$
$0.8)>$ $0.8)>$

$\mathbf{L}=<(0.3,0.5,0.4),(0.1,0.2,0.2),(0.7,0.5$, $\mathbf{M}=<(0.4,0.5,0.2),(0.3,0.3,0.1),(0.9,0.6$, $0.8)>$

$\mathbf{N}=<(0.3,0.5,0.4),(0.1,0.2,0.2),(0.7,0.5$ $0.8)>$

Ahora, Q, S, F, G, J, L, M y N son conjuntos $N^{*} b O$, pero no son conjuntos neutrosóficos abiertos.

\section{Conjuntos neutrosóficos *b-cerrados en espacios topológicos neutrosóficos}

En esta sección, se introduce la noción de conjunto neutrosófico $* b$-cerrado y se estudian sus propiedades.

Teorema 4.1 Un subconjunto $B$ en un NTS Y es un conjunto $N C S$ si y solo si $N \operatorname{Int}(N C l(B)) \mathrm{U} \mathrm{Cl}$ (NInt $(\mathrm{B})) \subseteq \mathrm{B}$.

Demostración: La demostración se sigue directamente de la definición 3.1.

Proposición 4.2 Sea $(\mathrm{Y}, \tau)$ un NTS y B un subconjunto neutrosófico de Y. Entonces, B es un conjunto $N^{*} b C$ si y solo si C (B) es un conjunto $N^{*} b O$ en $\mathrm{Y}$.

Teorema 4.3 Sea $(\mathrm{Y}, \tau)$ un NTS. Entonces, la intersección de dos $N^{*} b C$ es un conjunto $N^{*} b C$ en la NTS Y.

Demostración: La demostración se sigue directamente del teorema 3.2 y aplicando las leyes D’ Morgan.

Teorema 4.5 Sea $\left\{\mathrm{B}_{\alpha}\right\}_{\alpha_{\epsilon} \in}$ una colección de conjuntos $\mathrm{N}^{*} \mathrm{bC}$ en un NTS Y. Entonces, $\cap_{\mathrm{\alpha}_{\epsilon \in}} \mathrm{B}_{\mathrm{\alpha}}$ es un conjunto $\mathrm{N}^{*} \mathrm{bC}$ en $\mathrm{Y}$.

Demostración: La demostración se sigue directamente del teorema 4.3.

Observación 4.6 La unión de dos conjuntos $N^{*} b C$ no necesariamente es un conjunto $N^{*} b C$ en Y como se puede ver en el siguiente ejemplo.

Ejemplo 4.7 Sea $\mathrm{Y}=\{1\} \mathrm{y}$

$$
\begin{aligned}
& \mathrm{Q}=<(1,0.5,0.7)> \\
& \mathrm{W}=\langle(0,0.6,0.2)> \\
& \mathrm{E}=\langle(1,0.9,0.2)> \\
& \mathrm{R}=\langle(0,0.3,0.7)>
\end{aligned}
$$


Entonces $\tau=\{0 \mathrm{~N}, \mathrm{Q}, \mathrm{W}, \mathrm{E}, \mathrm{R}, 1 \mathrm{~N}\}$ es un NTS sobre $\mathrm{Y}$. Ahora, definamos los siguientes conjuntos $N^{*} b C$ como $\mathrm{P} 1=<(0.4,0.5,1)>$ y $\mathrm{P} 2=<(0.2,0$, $0.8)>$. Podemos ver que P1 U P2 $=<(0.4,0.5,0.8)$ $>$ no es un conjunto $\mathrm{N}^{*}$ bC en $\mathrm{Y}$.

Teorema 4.8 Todo conjunto neutrosófico cerrado en la NTS X es un conjunto $\mathrm{N}^{*}$ bC en $\mathrm{Y}$.

Demostración: La demostración se sigue directamente de las definiciones.

Observación 4.9 El reciproco del teorema anterior no se cumple de manera general como se puede ver en el siguiente ejemplo.

Ejemplo 4.10 Sea $\mathrm{Y}=\{q, w, e\}$ con $\tau=\left\{0_{\mathrm{N}}, \mathrm{Q}\right.$, $\left.\mathrm{W}, 1_{\mathrm{N}}\right\}$ y $\mathrm{C}(\tau)=\left\{1_{\mathrm{N}}, \mathrm{E}, \mathrm{R}, 0 \mathrm{~N}\right\}$ donde

$$
\mathrm{Q}=<(0.5,0.5,0.3),(0.1,0.1,0.9),(1,0.9,0.4)>
$$
$0.8)>$

$\mathrm{W}=<(0,0.3,0.1),(0.1,0.4,0.9),(0.5,0.8$, 1) $>$

$$
\mathrm{E}=<(0.3,0.1,0.5),(0.9,0.5,0.1),(0.4,0.9,
$$
$0.5)>$.

$$
\mathrm{R}=<(0.7,0.7,0),(0.9,0.4,0.1),(0.8,0.2,
$$

$$
\mathrm{T}=<(0.2,0.6,0.9),(0,0.9,0.9),(0.3,0.1,1)>.
$$

Aquí, los conjuntos $N^{*} b C$ son E, R y T. Además, T es un conjunto $N^{*} b C$ pero no es un conjunto neutrosófico cerrado.

\section{5. *b-interior $\mathbf{y} * b$-clausura es espacios topológicos neutrosóficos}

En esta sección, presentamos los operadores neutrosóficos *b-interior y *b-clausura y sus propiedades en un espacio topológico neutrosófico.
Definición 5.1 Sea $(Y, \tau)$ un NTS. Entonces, para un subconjunto neutrosófico A de $\mathrm{Y}$, neutrosófico *b-interior de $\mathrm{B}\left(\mathrm{N}^{*} \mathrm{~b}\right.$ Int $\left.(\mathrm{B})\right)$ es la unión de todos los conjuntos neutrosóficos $* b$-abiertos de $\mathrm{Y}$ contenidos en B. Es decir, $N^{*} b \operatorname{Int}(\mathrm{B})=\mathrm{U}\{\mathrm{W}$ : W es un conjunto $N^{*} b O$ en $\mathrm{Y}$ y $\left.\mathrm{W} \subseteq \mathrm{B}\right\}$

Proposición 5.2 Sea $(\mathrm{Y}, \tau)$ un NTS. Entonces, para cualquieras conjuntos neutrosóficos $B$ y $D$ de un NTS Y. Entonces, los siguientes enunciados se satisfacen:

(i) $N^{*} b \operatorname{lnt}(B) \subseteq B$

(ii) $B$ es un conjunto $N^{*} b O$ en $Y \Leftrightarrow N^{*} b \operatorname{lnt}(B)=B$

(iii) $N^{*} b \operatorname{lnt}\left(N^{*} b \operatorname{lnt}(B)\right)=N^{*} b \operatorname{lnt}(B)$

(iv) Si $B \subseteq D$ entonces $N^{*} b \operatorname{lnt}(B) \subseteq N^{*} b \operatorname{lnt}(D)$

\section{Demostración:}

(i) Es consecuencia directa de la definición 5.1.

(ii) Sea $B$ un conjunto $N^{*} b 0$ en $Y$. Entonces, $B \subseteq N^{*} b \operatorname{lnt}(B)$. De (i) tenemos que $B=N^{*} b$ Int (N). Recíprocamente, consideremos que $B=N^{*} b$ Int $(B)$. De la definición $5.1, B$ es un conjunto $N^{*} b 0$ en $Y$.

(iii) De (ii), tenemos $N^{*} b \operatorname{lnt}\left(N^{*} b \operatorname{lnt}(B)\right)=N^{*} b \operatorname{lnt}(B)$.

(iv) Dado que $B \subseteq D$, de (i), $N^{*} b \operatorname{lnt}(B) \subseteq B \subseteq D$. Aśi, $N^{*} b \operatorname{lnt}(B) \subseteq D$. De (iii), tenemos que

$N^{*} b \operatorname{lnt}\left(N^{*} b \operatorname{lnt}(B)\right) \subseteq N^{*} b \operatorname{lnt}(D)$. Por lo tanto, $N^{*} b \operatorname{lnt}(B) \subseteq N^{*} b \operatorname{lnt}(D)$.

Teorema 5.3 Sea $(\mathrm{Y}, \tau)$ un NTS. Entonces, para cualquieras conjuntos neutrosóficos $B$ y $D$ de un NTS, los siguientes enunciados se satisfacen:

Entonces $(\mathrm{X}, \tau)$ es un NTS. Ahora, consideremos las NSS $\mathrm{T}=\langle(0.7,0.6,0.1),(0.7,0.6,0.1)$, $(0.9,0.5,0)\rangle$ y $\mathrm{Y}=\langle(0.4,0.6,0.1),(0.5,0.7,0.2),(1,0.7,0.1)\rangle$. Entonces, $N^{*} b \operatorname{Int}(\mathrm{T})=\mathrm{R}$ y $N^{*} b \operatorname{Int}(\mathrm{Y})=\mathrm{R}$. Esto implica que $N^{*} b \operatorname{Int}(\mathrm{T}) \cup N^{*} b \operatorname{Int}(\mathrm{Y})=\mathrm{R}$. Ahora, $\mathrm{T} \cup \mathrm{Y}=\langle(0.7,0.6$, $0.1),(0.7,0.7,0.1),(1,0.7,0)\rangle$, de esto se sigue que $N^{*} b \operatorname{Int}(\mathrm{T} \cup \mathrm{Y})=\mathrm{W}$. Entonces, $N^{*} b$ Int 


\section{Demostración:}

(i) Dado que $B \cap D \subseteq B y B \cap D \subseteq D$, por la proposición 5.2 (iv), $N^{*} b \operatorname{lnt}(B \cap D) \subseteq N^{*} b \operatorname{lnt}(B)$ y $N^{*} b \operatorname{Int}(B \cap D) \subseteq N^{*} b \operatorname{lnt}(D)$. Esto implica que $N^{*} b \operatorname{Int}(B \cap D) \subseteq N^{*} b \operatorname{lnt}(B) \cap N^{*} b \operatorname{lnt}$ (D)(1). Por la proposición $5.2(i), N^{*} b \operatorname{lnt}(B) \subseteq B$ y $N^{*} b \operatorname{lnt}(D) \subseteq D$. Esto implica que $N^{*} b \operatorname{lnt}$ $(B) \cap N^{*} b \operatorname{lnt}(D) \subseteq B \cap D$. Ahora, aplicando la proposición 5.2 (iv), $N^{*} b$ Int $\left(\left(N^{*} b \operatorname{lnt}(B) \cap\right.\right.$ $\left.N^{*} b \operatorname{lnt}(D)\right) \subseteq N^{*} b \operatorname{lnt}(B \cap D)$. De $(1), N^{*} b \operatorname{lnt}\left(N^{*} b \operatorname{lnt}(B)\right) \cap N^{*} b \operatorname{lnt}\left(N^{*} b \operatorname{lnt}(D)\right) \subseteq N^{*} b \operatorname{lnt}(B$ $\cap D)$. De la proposición 5.2 (iii), $N^{*} b \operatorname{lnt}(B) \cap N^{*} b \operatorname{lnt}(D) \subseteq N^{*} b \operatorname{lnt}(B \cap D)(2)$. De (1) y (2), concluimos que $N^{*} b \operatorname{lnt}(B \cap D)=N S \operatorname{Int}(B) \cap N S \operatorname{lnt}(D)$.

(ii) Dado que $B \subseteq B \cup D y D \subseteq B \cup D$, de la proposición 5.2 (iv), $N^{*} b \operatorname{lnt}(B) \subseteq N^{*} b \operatorname{lnt}(B \cup D)$ and $N^{*} b \operatorname{lnt}(D) \subseteq N^{*} b \operatorname{lnt}(B \cup D)$. Esto implica que $N^{*} b \operatorname{lnt}(B) \cup N^{*} b \operatorname{lnt}(D) \subseteq N^{*} b \operatorname{lnt}(B \cup$ D).

El siguiente ejemplo muestra que la igualdad en el teorema 5.3 no necesariamente es verdadera.

Ejemplo 5.4 Sea $X=\{q, w, e\}$ y $\tau=\left\{0_{N}, Q, W\right.$, $\left.\mathrm{E}, \mathrm{R}, 1_{\mathrm{N}}\right\}$ donde

$\mathrm{Q}=<(0.4,0.7,0.1),(0.5,0.6,0.2),(0.9,0.7$,

$\mathrm{W}=<(0.4,0.6,0.1),(0.7,0.7,0.2),(0.9,0.5$, $0.1)>$,

$\mathrm{E}=<(0.4,0.7,0.1),(0.7,0.7,0.2),(0.9,0.7$, $0.1)>$

$\mathrm{R}=<(0.4,0.6,0.1),(0.5,0.6,0.2),(0.9,0.5$, $0.3)>$.

Entonces $(\mathrm{X}, \tau)$ es un NTS. Ahora, consideremos las NSS T $=\langle(0.7,0.6,0.1),(0.7,0.6,0.1)$, $(0.9,0.5,0)\rangle$ y $\mathrm{Y}=\langle(0.4,0.6,0.1),(0.5,0.7,0.2),(1,0.7,0.1)\rangle$. Entonces, $N^{*} b \operatorname{Int}(\mathrm{T})=\mathrm{R}$ y $N^{*} b \operatorname{Int}(\mathrm{Y})=\mathrm{R}$. Esto implica que $N^{*} b \operatorname{Int}(\mathrm{T}) \cup N^{*} b \operatorname{Int}(\mathrm{Y})=\mathrm{R}$. Ahora, $\mathrm{T} \cup \mathrm{Y}=\langle(0.7,0.6$, $0.1),(0.7,0.7,0.1),(1,0.7,0)\rangle$, de esto se sigue que $N^{*} b \operatorname{Int}(\mathrm{T} \cup \mathrm{Y})=\mathrm{W}$. Entonces, $N^{*} b$ Int $(\mathrm{T} \cup \mathrm{Y}) \nsubseteq N^{*} b \operatorname{Int}(\mathrm{T}) \cup N^{*} b \operatorname{Int}(\mathrm{Y})$.

Definición 5.5 Sea $(Y, \tau)$ un NTS. Entonces, para-cualquier subconjunto neutrosófico $\mathrm{B}$ de $\mathrm{Y}$, neutrosófica *b-clausura de $\mathrm{B}(\mathrm{N} * \mathrm{bCl}(\mathrm{B}))$ es la intersección de todos los conjuntos neutrosóficos *b-cerrados de $\mathrm{Y}$ contenidos en $\mathrm{B}$. Es decir, $\mathrm{N} * \mathrm{~b} \mathrm{Cl}$ (B) $=\cap\left\{\mathrm{W}: \mathrm{W}\right.$ es un conjunto neutrosófico $\mathrm{N}^{*} \mathrm{~b} \mathrm{C}$ en $\mathrm{Y} \mathrm{y} \mathrm{W} \supset \mathrm{B}$ \}.
Proposición 5.6 Sea $(Y, \tau)$ un NTS. Entonces, para cualquier subconjunto neutrosófico B de $\mathrm{Y}$, los siguientes enunciados se satisfacen:

(i) $\mathrm{C}(\mathrm{N} * \mathrm{~b}$ Int (B) $)=\mathrm{N}^{*} \mathrm{~b} \mathrm{Cl}(\mathrm{C}(\mathrm{B}))$,

(ii) $\mathrm{C}(\mathrm{N} * \mathrm{~b} \mathrm{Cl}(\mathrm{B}))=\mathrm{N} * \mathrm{~b} \operatorname{Int}(\mathrm{C}(\mathrm{B}))$.

\section{Demostración:}

(i) De la definición $5.1, X^{*} b \operatorname{Int}(B)=\cup\left\{\mathrm{W}\right.$ : W es un conjunto $\mathrm{N}^{\mathrm{x}} \mathrm{b} 0$ en $Y$ y $\left.\mathrm{W} \subseteq B\right\}$. Tomando el complemento en ambos lados de las igualdades, $\mathrm{C}\left(\mathrm{N}^{*} b \operatorname{Int}(\mathrm{B})\right)=\mathrm{C}(\cup\{\mathrm{W}: \mathrm{W}$ es un conjunto $N^{*} b 0$ en $\mathrm{Y}$ y $\left.\left.\mathrm{W} \subseteq B\right\}\right)=\cap\left\{\mathrm{C}(\mathrm{W}): \mathrm{C}(\mathrm{W})\right.$ es un conjunto $N^{*} b C$ en $\mathrm{Y}$ y $\mathrm{C}$ $(B) \subseteq \mathrm{C}(\mathrm{W})\}$. Reemplazando $\mathrm{C}(\mathrm{W})$ por $\mathrm{R}$, tenemos que $\mathrm{C}\left(N^{*} b \operatorname{Int}(B)\right)=\cap\{\mathrm{R}: \mathrm{R}$ es un conjunto $N^{*} b C$ en $Y$ y $\left.\mathrm{R}_{\supseteq} \mathrm{C}(B)\right\}$. De la definición $5.5, \mathrm{C}\left(N^{*} b \operatorname{Int}(B)\right)=N^{*} b C l(\mathrm{C}(B))$.

(ii) De (i), tenemos que $\mathrm{C}\left(N^{*} b \operatorname{Int}(\mathrm{C}(B))\right)=N^{*} b \mathrm{Cl}((\mathrm{C}(B)))=N^{*} b \mathrm{Cl}(B)$. Tomando el complemento en ambos lados, tenemos $N^{*} b \operatorname{Int}(\mathrm{C}(B))=\mathrm{C}\left(N^{*} b C l(B)\right)$.

Proposición 5.7 Sea $(\mathrm{Y}, \tau)$ un NTS. Entonces, para cualquieras subconjuntos neutrosóficos $B$ y $D$ de un NTS Y. Entonces, los siguientes enunciados se satisfacen:

(i) $B \subseteq N^{*} b C l(B)$

(ii) $B$ es un conjunto $N^{*} b C$ en $Y \Leftrightarrow N^{*} b C l(B)=B$

(iii) $N^{*} b C l\left(N^{*} b C l(B)\right)=N^{*} b C l(B)$

(iv) $S i B \subseteq D$ entonces $N^{*} b C l(B) \subseteq N^{*} b C l(D)$ 


\section{Demostración:}

(i) Se sigue directamente de la definición 5.5 .

(ii) Sea $B$ un conjunto neutrosófico $N^{*} b C$ en $Y$. De la definición 4.2, $C$ (B) es un conjunto $N^{*} b 0$ en Y. De la proposición 5.6 (ii), $N^{*} b \operatorname{Int}(\mathrm{C}(B))=\mathrm{C}(B) \Leftrightarrow \mathrm{C}\left(N^{*} b C l(B)\right)$ $=\mathrm{C}(B) \Leftrightarrow N^{*} b C l(B)=B$.

(iii) De (ii), tenemos $N^{*} b C l\left(N^{*} b C \mid(B)\right)=N^{*} b C l(A)$

(iv) Dado que $B \subseteq D, C(D) \subseteq C(B)$. De la Proposición 5.2 (iv), $N^{*} b \operatorname{lnt}(\mathrm{C}(D)) \subseteq N^{*} b \operatorname{lnt}$ (C

(B)). Tomando complemento en ambos lados, $\mathrm{C}\left(N^{*} b \operatorname{lnt}(\mathrm{C}(D))\right) \supseteq \mathrm{C}\left(N^{*} b \operatorname{lnt}(\mathrm{C}(B))\right)$. De la proposición 5.6 (ii), $N \mathrm{~N} b C l(B) \subseteq N b C l(D)$.

Proposición 5.8 Sea $(Y, \tau)$ un NTS. Entonces, para cualquieras subconjuntos neutrosóficos B y D de un NTS Y. Entonces, los siguientes enunciados se satisfacen:

(i) $N^{*} b C l(B \cup D)=N^{*} b C l(B) \cup N^{*} b C l(D)$

(ii) $N^{*} b C l(B \cap D) \subseteq N^{*} b C l(B) \cap N^{*} b C l(D)$.

\section{Demostración:}

(i) Dado que $N^{*} b C l(B \cup D)=N^{*} b C l(C(C(B \cup D)))$, de la proposición $5.6(i), N^{*} b C l(B \cup D)$ $=C\left(N^{*} b \operatorname{lnt}(C(B \cup D))\right)=C\left(N^{*} b \operatorname{lnt}(C(B) \cap C(D))\right)$. Utilizando la proposición $5.7(i), N^{*} b C l$ $(B \cup D)=C\left(N^{*} b \operatorname{lnt}(C(B)) \cap N^{*} b \operatorname{lnt}(C(D))\right)=C\left(N^{*} b \operatorname{lnt}(C(B)) \cup C\left(N^{*} b \operatorname{lnt}(C(D))\right)\right.$. De la proposición $5.6(i), N^{*} b C l(B \cup D)=N^{*} b C l(C(C(B))) \cup N^{*} b C l(C(C(D)))=N^{*} b C l(B) \cup N^{*} b C l$ (D).

(ii) Dado que $B \cap D \subseteq B$ y $B \cap D \subseteq D$, de la proposición 5.7 (iv), $N^{*} b C l(B \cap D) \subseteq N^{*} b C l(B)$ y $N^{*} b C l(B \cap D) \subseteq N^{*} b C l(D)$. Esto implica que $N^{*} b C l(B \cap D) \subseteq N^{*} b C l(B) \cap N^{*} b C l(D)$.

El siguiente ejemplo muestra que la igualdad de la proposición 5.8 no se satisface generalmente.

Ejemplo 6.6 Sea $Y=\{a, b, c\}$ con $\tau=\{0 N, A$, $\mathrm{B}, \mathrm{C}, \mathrm{D}, 1 \mathrm{~N}\}$ y $\mathrm{C}(\tau)=\{1 \mathrm{~N}, \mathrm{E}, \mathrm{F}, \mathrm{G}, \mathrm{H}, 0 \mathrm{~N}\}$ donde $0.2)>$

$$
\mathrm{A}=<(0.5,0.6,0.1),(0.6,0.7,0.1),(0.9,0.5,
$$
$0.3)>$
$0.3)>$

$$
\mathrm{C}=<(0.4,0.5,0.2),(0.6,0.6,0.3),(0.9,0.5,
$$

$$
\mathrm{D}=<(0.5,0.6,0.1),(0.8,0.7,0.1),(0.9,0.7,
$$
$0.2)>$

$$
\mathrm{E}=<(0.1,0.4,0.5),(0.1,0.3,0.6),(0.2,0.5,
$$
$0.9)>$,

$$
\mathrm{F}=<(0.2,0.5,0.4),(0.3,0.4,0.8),(0.3,0.3,
$$
$0.9)>$,

$\mathrm{G}=<(0.2,0.5,0.4),(0.3,0.4,0.6),(0.3,0.5$ $0.9)>$,

$\mathrm{H}=<(0.1,0.4,0.5),(0.1,0.3,0.8),(0.2,0.3$, $0.9)>$.

$N^{*} b s$

Entonces $(Y, \tau)$ es un NTS. Ahora considere los

$\mathrm{I}=\langle(0.1,0.2,0.5),(0.2,0.3,0.7),(0.3,0.3,1)\rangle \mathrm{y} \mathrm{J}=\langle(0.2,0.4,0.8),(0.1,0.2,0.8),(0.2,0.5$, 0.9)). Entonces, $N^{*} b C l(\mathrm{I})=\mathrm{G}$ y $N^{*} b C l(\mathrm{~J})=\mathrm{G}$. Esto implica que $N^{*} b C l(\mathrm{I}) \cap N^{*} b C l(\mathrm{~J})=\mathrm{G}$. Ahora, $I \cap \mathrm{J}=\langle(0.1,0.2,0.8),(0.1,0.2,0.8),(0.2,0.3,1)\rangle$, de esto se sigue que $\mathrm{N}^{*} b \mathrm{Cl}(\mathrm{I} \cap$ $\mathrm{J})=$ H. Entonces, $N^{*} b C l(\mathrm{I}) \cap N^{*} b C l(\mathrm{~J}) \nsubseteq N^{*} b C l(\mathrm{I} \cap \mathrm{J})$.

\section{Conclusión}

En este artículo se ha introducido la noción de conjuntos neutrosóficos $* b$-abiertos en espacios topológicos neutrosóficos. Las nociones aquí presentadas se pueden generalizar en diferentes ramas de los conjuntos neutrosóficos. Adicionalmente, se pueden encontrar algunas relaciones entre conjuntos abiertos neutrosóficos definidos en la literatura.

\section{Referencias}

Chang, C. (1986). Fuzzy Topological Spaces. J. Math. Anal. Appl. 24, 182-190. https://doi. org/10.1016/0022-247X(68)90057-7

Coker, D. (1997). An introduction to intuitionistic fuzzy topological spaces, Fuzzy Sets and Systems. 88, 81-89. https://doi.org/10.1016/ S0165-0114(96)00076-0

Das, S., Das, R., Granados, C., y Mukherjee, A. (2021). Pentapartitioned neutrosophic Q-ideals of Q-algebra, Neutrosophic Sets and Systems. 41, 
52-63. http://doi.org/10.5281/zenodo.4625678

Das, S., y Tripathy, B. (2020). Pairwise neutrosophic b-open set in neutrosophic bitopological spaces, Neutrosophic Sets and Systems. 38, 135-144. http://doi.org/10.5281/zenodo.4300463

Granados, C., y Dhital, A. (2021). Statistical convergence of double sequences in neutrosophic normed spaces, Neutrosophic Sets and Systems. 42, 333-344. http://doi.org/10.5281/ zenodo.4914796

Granados, C., y Dhital, A. (2021). New results on pythagorean neutrosophic open sets in pythagorean neutrosophic topological spaces, Neutrosophic Sets and Systems. 43, 12-23. http://doi.org/10.5281/zenodo.4920166

Granados, C. (2020). Un nuevo estudio de los conjuntos supra neutrosophic crisp, Revista Facultad de Ciencias Básicas. 16(2), 65-75. https://doi.org/10.18359/rfcb.5408

Granados, C., y Padilla, I. (2021). El aprendizaje gráfico de la recta tangente a través de la modelación de las secciones cónicas utilizando Geogebra, Revista Científica. 40(1), 118-132. https://revisthttps//doi. org/10.14483/23448350.16137

Hanafy, M. (2003). Completely continuous functions in intutionistic fuzzy topological spaces, Czechoslovak Mathematics journal. 53(4), 793-803. https://doi.org/10.1023/ B:CMAJ.0000024523.64828.31

Karaaslan, F. (2015). Neutrosophic soft sets with applications in decision making, International Journal of Information Science and Intelligent System. 4(2), 1-20. http://doi.org/10.5281/ zenodo. 23151

Salama, A., y Alblowi, S. (2012), Generalized
Neutrosophic Set and Generalized Neutrousophic Topological Spaces. Journal computer Sci. Engineering. 2(7)., 129-132. http://doi.org/10.5923/j.computer.20120207.01

Salama, A., y Alblowi, S. (2012). Neutrosophic set and neutrosophic topological space, ISOR J. mathematics. 3(4), 31-35. Disponible en: http://dl.icdst.org/pdfs/ files3/228e3c837770f2e14afbb606c3140d0c. pdf

Smarandache, F. (2002). Neutrosophy and Neutrosophic Logic, First International Conference on Neutrosophy, Neutrosophic Logic, Set, Probability, and Statistics University of New Mexico, Gallup, NM 87301, USA. Disponible en: https://arxiv.org/ftp/math/ papers/0306/0306384.pdf

Smaradache, F. (2010). Neutrosophic Set: A Generalization of Intuitionistic Fuzzy set, Journal of Defense Resourses Management. 1, 107-116. Disponible en: http://fs.unm.edu/ ScArt/NeutrosophicSetGeneralization.pdf

Smarandache, F. (2020). Neutroalgebra is a generalization of partial algebra, International Journal of Neutrosophic Science. 2(1), 8-17. Disponible en: https://www.researchgate.net/ publication/339883249_NeutroAlgebra_is_a_ Generalization_of_Partial_Algebra

Vergel, M, Virginia, R., y Rincón, O. (2016). Influencia de curso precálculo y actividades de apoyo institucional en desarrollo de competencias y creencias en matemáticas, Eco Matemático.7(1), 33-47. https://doi. org/10.22463/17948231.1099

Zadeh, L. (1965). Fuzzy Sets, Inform and Control. 8, 338- 353. https://doi.org/10.1016/S00199958(65)90241-X 
Zamora, J., Ramírez, J., y Delgado, F. (2020). Uso de herramientas tecnológicas y su impacto en el rendimiento en el curso de cálculo II de la Universidad Nacional, Eco Matemático. 11(1), 6-17. Disponible en: https://www. researchgate.net/publication/343812944_Uso_ de_herramientas_tecnologicas_y_su_impacto_ en_el_rendimiento_en_el_curso_de_Calculo_ II_de_la_Universidad_ 